of the anatomical nock except that a larger axillary pad is indicated.

Fractures of the shaft are treated by malleable iron fracture splints, which can be bent to cover in the shoulderjoint and thus produce fixation.

Al fractures in the region of the elbow-joint, with the exc stion of the olecranon, are treated, after adequate and

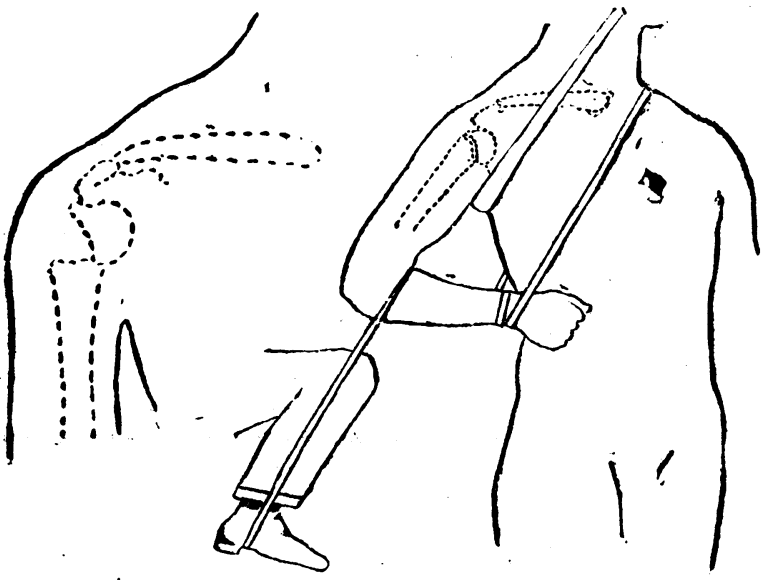

FIG. 5-Thomas's method for reduction of impaction in fracture of the surgicel neck of the humerus.

complete reduction, in the Jones or fully-flexed position. This position cannot be obtained unless reduction is complete. Whether the fracture is supracondylar, T-shaped, or intercondylar, one must reduce by traction-extension, supination, and hyperflexion, and, if the latter cannot be obtained, the routine must be repeated until it is, and the arm must then be kept in this position at rest for at least three to four weeks. A warning must be given against passive movements after the arm is being gradually brought down, otherwise traumatic arthritis and consequent stiffness of the elbow ensues. As an empiricism it is advisable to let the wrist sling down a little every week until the arm is at a right angle at the end of the sixth week. After the fourth week the patient can use the arm in the sling with moderation, and mussage may be given as an adjunct to treatment, but forcible passive movement is absolutely contraindicated. For this type of fracture the rectangular internal splint should be abolished.

Fractures of the head and neck of the radius must be fixed in full supination, otherwise limitation of this movement will certainly result. Fractures of the shaft of both bones require consideration of two important points: first, the shaft of the ulna must be kept straight, and secondly, the curve of the radius must be conserved as the
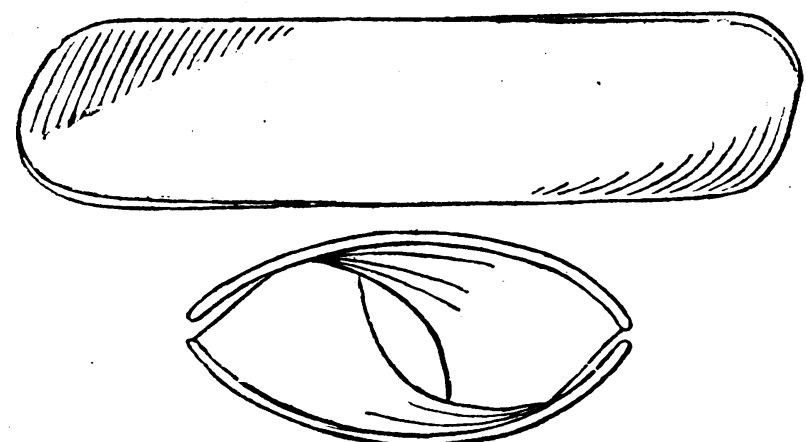

FIG. 6. Splints twisted for the treatment of Colles's fracture.

whole length of the posterior border of the ulna is straight, and on this the curved radius rotates like a bucket handle. There must be no lateral pressure on the middle of the radius, otherwise synostosis may ensue. In the position of supination the bones are farthest from each other, and so we should treat all our shaft fractures in this position. Nearly all neglected fractures of the forearm show limitation of supination, whereas pronation is not defective.

Colles's fracture requires complete and adequate reduction before splinting takes place. The Jones method is preferable to the hand-shaking plan, because the force is applied directly to the bone instead of working through the carpal bones. Twisted fracture splints (Fig. 6), adequately padded to keep the replaced fragments in an over-corrected position and the hand in ulnar deviation, are applied and remain for four weeks, move. ments of fingers and thumb being allorred from the first.

REFERENCE.

1 BRItish Medical JOURNAL, 1912, ii, p. 1505.

\section{Further Gbartrations}

\section{THE ETIOLOGY OF ECLAMPSIA AND THE PRE-ECLAMPTIC STATE.}

\section{$\mathrm{BX}$}

JAMES YOUNG, D.S.O., M.D., F.R.C.S., AESISTANT PHYSICIAN, ROYAL MIATERNITY HOBPITAL; TOTOR IN GYNAECOLOGY, UNIVERSITY OF EDINBURGH ;

DOUGLAS A. MILLER, M.B., CH.B. ASSISTAYT TO THE PROFESSOR OF WIDWIFERY ; RESEARCH SCHOLAR, VNIVERSITY OF EDINBCRGH.

THIs paper* contains confirmation of an explanation of eclampsia which one of us published in 1914. It is based on a study, carried out at the Royal Maternity Hospital, Edinburgh, of 40 cases of toxaemia and associated conditions. The original investigations professed to shorv that the eclamptic and the pre-eclamptic toxaemia is dependent on a placental necrosis and they culminated in the experimental reproduction in animals of a disease closely simulating the classical picture of eclampsia. The first observations were concerned with a study of the pathology and with experiment. We propose now to gire a third link of proof-the clinical.

Eclampsia and the pre-eclamptic state of toxaemia are due to the circulation in the mother's blood of a specific substance or specific substances, that have, as their dominating effects, (1) degenerative changes in the kidney and albuminuria, (2) characteristic necrotic lesions in the liver, and (3) convulsions. The symptom.pathological complex is found only in pregnancy, and it is therefore dependent, in some way or other, on the child or placenta or both, and its occurrence in cases of hydatid mole, where there is no foetus, points to the placental elements as the source of the poison.

Placental disease of the nature of infarction is common in eclampsia and pre-eclampsia. In fact, the typical case of long-standing albuminuria of pregnancy slows multiple pale areas of necrosis in the placenta. On the other hand, many cases of fulminating eclampsia show no obvious change in the placenta, and the natural inference for long was that, where infarction is present, it is only of secondary importance. If related to the toxaemic state at all-and related it must be, everyone who has studied the subject admits-it is considered as more the result of the toxaemia than the cause.

What first directed our attention to the subject was the discovery that, although obvious placental disease might be absent in the placenta of fulminating eclampsia, which ends in rapid labour or death, in the other, less virulent type, in which some days or even weelss elapse between the seizure and the birth of the placenta, there is always massive necrosis visible to the nalked eye. We have seen no exception to this. Its recognition threw a flood of new light on the subject. It showed that the degenerative change is always present in the placenta, but that it requires some days before it evolves into the form of ordinary nalied-eye infarction. As a matter of fact, the recognition of the real character of the change has made it possible of detection at an earlier period if it is looked for.

If these observations are correct they have a double significance. In the first place, they provide us with a tangible focus for the origin of a placental poison. If we except Veit's discarded theory of the deportation of chorionic villi into the blood stream, they offer such a focus for the first. time. In the second place, they

* Read before the Section of Obstetrics and Gynaecology of the 
indicate that, if the necrotic areas are the source of the eclamptic poison, this poison is produced during the early autolysis of the disintegration process, and whilst the structure of the placenta shows no, or only few, visible signs of change. By the time the process has progressed to the stage of ordinary naked-eye infarction the damage has been done.

Gradual piecemeal involvement of the placenta may produce in the end an extensive infarction consisting of small isolated necrotic areas of differing ages and wiih no symptoms except a toxaemia, which may never at any one time be of a virulent nature, and may, in fact, escape notice. A sudden single massive placental death may, on the other hand, kill before there is even a trace of visible infarction.

At the time of our original investigations in 1914 the idea that products eliminated from the disintegration of tissues at such an early stage could be toxic was a new one in pathology. There were no analogies from other branches of medicine to guide and encourage us. That the impressions formed on the basis of our anatomical studies were, however, well founded was borne out in a very complete degree by the results of our experimental work. We showed that autolysis of the human placenta increased its toxicity to the lower animals, and, moreover, we demonstrated that the early autolytic materials are more toxic than those present later; it seemed as if the early toxic matter was labile, and, if left, became broken up into less noxious elements, perhaps as the result of the continued action of the cell-enzymes. We claimed at that time to have reproduced in the lower animals, by the injection of the early autolytic products of the placenta, a disease exhibiting the classical features of human eclampsia. At that time, as we have said, the conceptions underlying our belief were new in pathology. Since then, however, it has been recognized that similar factors operate in other diseased conditions, and so exactly have these new developments followed the lines laid down in our original investigations that they provide, by analogy, strong proof of our thesis.

Wo would specially direct attention to the investigations on the origin of shock (Quénu, Delbet, etc.), and more especially to the experimental reproduction of toxaemia by the injection of the products of damaged muscle. Delbet has demonstrated by an extensive and convincing series of experiments that the fission products of muscle are toxic even in the very earliest stages of disintegration. The results have been confirmed by others. A very interesting discovery made during these experiments was that the animal might die immediately or within a short time from an apparent intoxication of the nervous system. On the other hand, it might live for hours or a day or more, and thon die from damage to the liver or suprarenal capsules. As Delbet says: "Ces morts retardees sont dues à des altérations du foie et des capsules surrénales." The application of these findings to eclampsia will become apparent in the latter part of this paper.

Our original investigations were arrested by the war. Since they were begun again, during the past year, we have confirmed our anatomical and experimental results. But here we desire more particularly to adduce another and different line of proof based on clinical observations. Before doing so perhaps it will be well to recapitulate briefly our previous observations on the pathology and etiology of placental infarction. Fortunately they are capable of easy demonstration.

\section{Pathology of Infarction.}

The pathological clianges in an infarcted area of placenta are:

1. Coagulation in the intervillous spaces. This, as we shall see, is due to a blockage of the corresponding maternal vessels.

2. Congestion of the vessels in the foetal villi. This is early and characteristic. The expansion of the vessels causes a swelling of the villi, which become closely packed together to form a solid block resting against the decidual surface, and sharply differentiated from the sur: roukding spongy placental substance. This hepatization is not entirely due the the turgescence of the individual villi. It is due also, and probably largely, to the tension of the environing placenta which presses in on an. aroa in which the circulation is arrested. The infarcted block is thus really an area of collapsed placental tissue.

3. Necrosis.-During the necrosis the villi undergo disintegration of their cellular elements until in the end they are represented by mere glost-like outlines. During the process the infarct passes through a series of colour changes. - In the beginning it is purple or red, then it becomes chocolate-co!oured, then brown, and, with the disappearance of the haemoglobin, the colour eventually of an old infarct is yellow or white. The entire evolution of change requires a considerable number of days.

The naked-eye recognition of infarction is easy when it is fully established; the pale infarct is so distinctive that it forms a prominent feature of the cut section of the placenta. 'The red or purple infarct is more likely to escape notice as its colour may not differ greatly from the surrounding placenta. Except in the early stages, however, it is solid, and is therefore sharply cut off from the surrounding soft and spongy placenta. In the inter. mediate stages an infarct may be red in one part and pale in another. In many of the early cases the infarction can only be recognized when the cut section is gently washed with running water. This rinses the intervillous blood out of the lealthy parts of the placenta, which become pale, and thus bring into sharp relief the purple blocks of infarcted tissue, in wlich the blood is clotted and is therefore not remored by the washing.

\section{Etiology of Infarction.}

As we have shown in another communication, the underlying factor is an interference with the maternal blood supply. 'This may be due to simple mechanical factors'for example, separation of the placenta from the uterus by trauma or retroplacental bleeding. These are the circum. stances that commonly accompany a case of accidental haemorrhage. Another common condition of a somewhat similar nature is placenta praevia, the bearing of which on the questions under discussion will be mentioned later.

In many instances there is no evidence of placental separation or of retroplacental bleeding, and the local impairment of the blood supply that ends in placental necrosis is due to thrombosis. We have noted this frequently, and we believe it to be an important cause of infarction.

Accidental Haemorrhage and Toxaemia.

Retroplacental haemorrhage is a common cause of placental degeneration, and it possesses a special interest for us on account of the frequency with which it is associated with toxaemia. The frequency of albuminuria in accidental haemorrhage is given by different authors in figures varying from 30 per cent. to 80 per cent. or over. Our own ratio is nineteen times in a series of twenty-one cases-that is, 90 per cent.

It is often urged that the toxaemia is the cause of the bleeding, and one can see low the discovery of the common association of the two conditions should have given rise to this belief. But we would direct notice to some considerations that biing the validity of the common interpretation under question.

In the first place, accidental haemorrhage is relatively much rarer in primiparae than in multiparae, though toxaemia is specially prone to affect the former.

II.

In the second place, a study of the etiology of accidental haemorrhage shows that a severe strain or other mechanical factor may cause the condition in a woman who was apparently healthy up to that time. The importance of a purely mechanical fuctor is well brought out by the clinical study of our cases. In 4 out of a series of 21 cases the bleeding followed the performance of such severe exertion as the washing of clothes, or it succeeded an accident, such as falling downstairs. And in all these cases there was albuminuria. In two cases the albuminuria developed after bleeding; the inaportance of this will emerge later.

In most cases of accidental haemorrhage the bleeding occurs in the site of least resistance-namely, in the displacèable retroplacental area. In other cases; however, the bleeding is widespread, and, coincident with the retro. 
placental extravasation, there may be a deep infiltration of the muscular wall of the uterus and of the broad ligaments, \& condition which has been called "diffuse uteroplacental apoplesy." In our series there are two cases of this remarkablo condition. In the one the extravasation involved the whole uterus and the broad ligaments on both sides. In the other the bleeding was localized chiefly at the right cornu and the adjacent part of the tube and broad ligament. The appearances are exactly those that would be produced by a sudden blockage of the right ovarian vein. The intense and sudden congestion of the uterus which is produced in these cases resembles the change induced in a pedunculated ovarian or fibromyomatous tumour by an acute torsion of its pedicle.

What is the cause of the sudden blockage of the veins in the pelvis that alone can account for a backward pressure sufficient to tear open the vessels of the uterine wall? Is it a thrombosis or a kinking, or is it due to a sudden increase of the intra-abdominal pressure?

III.

A study of the placenta in cases of accidental haemor. rhage provides a third series of considerations in support of the theory which we are adrocating.

Accidental haomorrhage may be divided into two classes: (a) those in which the placenta shows little or no evidence of degeneration, and (b) those in which the placenta exhibits degeneration of the detached area or areas.

(a) Here sufficient time has not been allowed for the full development of infarction. This category includes ca:es in which the birth of the placenta follows quickly on the onset of the bleeding, and in it are numbered most cases of external bleeding. Toxaemia is inconspicnous, and if present is represented by a slight transient albuminuria.

(b) Here the placenta throughout the detached area shows the signs of early infarction. The placenta has been retained in the nterus for some time, and, whilst the one part of the organ has remained intact and healthy, the other part has become transformed into a mass of degeneration. The degree of hepatization present is dependent on the interval that has elapsed between the local bleeding and the birth of the placenta. This observation is one which we have made so often that we have removed it from the category of the occasional or the accidental. It is invariable.

We have seen one case in which nearly half of the placenta was in a state of early purple infarction. In other cases the bleeding has taken place over small areas - the so-called "retroplacental haematomata" -and the infarcted regions are correspondingly small. Between these two extremes there are all grades in the extent of the retroplacental bleeding, and therefore in the size of the subtending areas of infarction.

Toxaemia is a dominating feature in this class of case. The most severe toxaemias in our series of retroplacental haemorrhage belong to this class. It includes five cases of eclampsia.

IV.

Finally, that the toxaemia is secondary is shown by the fact that we have actually demonstrated that it develops subsequent to the haemorrhage. In two of our cases an examination of the urine within a few hours of the bleeding was negative, whereas at a later examination an albuminuria was revealed. An albuminuria develops so rapidly after the separation of the placente that it is usually present by the time the patient is admitted to hospital. In many cases, especially of external bleeding, the albumin may never be present except as a trace. It develops early, and in the great majority of instances it passes off early.

It will have become apparent that, if our observations are correct, in order that a toxaemia may develop it is necessary that the placenta must maintain its attachment to one part of the uterine wall. Only otherwise can it receive and pass into the maternal blood the products of the adjacent necrosis. The placenta is like a large bag, in which there is a free circulation of blood, and a necrotic area is thas like a poison-laden mass introduced directly into the maternal vessels.

A recognition of this fact accounts for the well known clinical observation that the toxaemia often ceases with the intrauterine death of the child and the complete separation of the placenta.
A suggestive analogy to the conditions which obtain in placental degeneration is supplied by the observations of Quénu in regard to shock. He showed that the application of a tourniquet to a shattered limb prevented the occur. rence of shock by blocking off the source of the disintegration products, whereas the removal of the touiniquet by re-establishing a communication with the systemic circulation was followed by toxaemic symptoms. These observations are comparable to ours-toxaemia from the placenta can only arise and be maintained if and so long as part of the placental circulation persists.

\section{Placenta Praevia and Toxaemia.}

It sometimes happens that, in placenta praevia, the detachment of the placenta commences some time before the delivery of the uterine contents. If our thesis be correct such cases should be associated with a toxaemia. We therefore decided to conduct an investigation along these lines.

Albuminuria is the earliest definite sign of a toxaemia that we possess, and in this investigation, as in the similar investigation of cases of accidental hsemorrhage, the presence of albumin in the urine was taken as the test. This sign is sufficient, but it is well known that a trace of albumin may be present without any other evidence whatever of a toxic process.

Toxaemia has, so far as we know, never been recognized as bearing any relation to placenta praevia, though a number of cases have been recorded in which low im. plantation of the placenta was combined with albuminuria. The concurrence has been, however, considered as a chance phenomenon. Recently Jardine and Kennedy reported a series of eleven cases of toxaemia so great as to lead to complete suppression of urine, and in two of these (that is, one in five and a half) there was placenta praevia. These conditions are each so rare that unless there is a possible mutual dependence they should occur together only once in several hundred thousand cases.

For this investigation, as for the similar investigation of the cases of accidental haemorrhage, a catheter specimen of urine was obtained as soon as possible after the admission of the patient to hospital, and also at subsequent intervals thereafter. In all the cases bleeding had commenced before their admission.

Our series consists of 7 cases of placenta praevia. Of these 2 had no albuminuria throughout. In 5 cases albuminuria was found. In 2 it increased from a trace at one examination to a heavy deposit at subsequent examinations. In 1 the alburninuria was absent at the first examinations and only developed later. In 3 of the patients the albuminuria was associated with other evidences of toxremia, oedema of the face, headache, etc. whilst in one of these cases the toxic symptoms were so great that her condition caused considerable anxiety.

This record is striking, and it is, we believe, a powerful confirmation of the argument we are attempting to prove. The series is, however, too small to warrant an y far-reaching conclusions, and we wish to avoid any such temptation. We would rather say that we welcome the opportunity of presenting this evidence, especially because it is of a nature that can be put to a ready test in any obstetric clinic. We shall be glad to hear the results of any others who make similar investigations.

The facts, however, raise a suspicion that in the past the toxaemia which apparently is liable to occur in placenta praevia has been overlooked and has been attributed to the collapse and the anaemia of haemorrhage. We may say that, as a control, we examined two cases of severe post-partum haomorrhage at intervals and in each case the urine gave negative findings. The changes in the detached praevia portion of the placenta are exactly those which we have described in the detached placenta of accidental haemorrhage. Where time has been allowed for their development the macroscopic and microscopic features of early infarction are found.

Other Causes of Placental Degeneration and Toxaemia.

In the preceding pages it has been shown that mechanical factors may cause a detachment of the placenta from the uterine wall, and, as in placenta praevia and in some cases of accidental haemorrhage, such a mechanical detachment is the cause of the de. generation that ends in toxaemia. 
It often happens, however, that in cases of ordinary infarction of the placenta the exact cause of the impairment of the maternal blood supply is difficult or impossible to discover by an anatomical examination. In some sucl instances there is a thrombosis in the decidual vessels, and we believe that this factor probably operates in a number of cases. But it is impossible of proof, for one cannot tell from the microscopic appearances whether the thrombosis has preceded or followed the necrosis.

So, in many cases of eclampsia and albuminuria in which definite placental disease is present, the exact origin of the blockage of the maternal vessels may not be evident. We have shown, we believe conclusively, that such a blockage can alone account for the placental changes. How, then, are we to explain it in the absence of such simple factors as are present always in placenta praevia and often in accidental haemorrhage? Do mechanical influences operate in these cases as well?

In this connexion we would again direct attention to one of our cases in which the retroplacental bleeding was associated with a haemorrhage into the uterine wall the tube, and the broad ligament in a way which we could only explain by involing a sudden strangulation of the right ovarian vein. In the other case of "diffuse uteroplacental apoplexy" the appearances again suggested a mechanical strangulation of the veins, but here of both ovarian and perhaps of the uterine veins as well. The conditions were similar bat more extensive.

We have referred to the remarkable and suggestive similarity between ateri showing these evidences of strangulation and an ovarian tumour or a pedunculated fibromyomatous tumour with a twisted pedicle. The conditions are so alike that it is almost impossible to escape the conviction that similar causes are operating.

If there are intra-abdominal influences, perlaps of the form of heightened tension, which can in their extreme form culminate in such a dramatic vascular change, it is almost certain that these same pressure factors act at other times in a less marked degree. It is known that, especially in primiparae, there are evidences of increased intra-abdominal tension-for example, swelling of the feet and legs and distension of the right ureter. Is it possible that similar agencies tend to cause venous stasis? In their extreme form this would result in complete strangulation, whilst in its more mild degree it would result in thrombosis of the veins of the retroplacental area or of the uterine wall.

Localized degeneration in the placenta must be due to blockage of the corresponding vessels. Of this we can be certain, but the altimate explanation must remain of a theoretical nature until we have a more intimate knowledge of the vascular conditions that obtain in the abdo. men and pelvis during pregnancy. Paramore maintains that he has actually demonstrated the existence of a heightened intra-abdominal pressure during pregnancy. We have not had an opportunity of carrying out investigations along these lines, but we have a strong belief that it is by such a research that we shall eventually explain the local necrosis of the placenta which underlies some, if not all, of the toxaemias of the end period of pregnancy.

\section{Sequence of Pathological Changes in Eclampsia :} Post-partum Eclampsia.

In eclampsia there are widespread evidences of a toxic process throughout the body of the mother, the most pro. minent of which are the damage to the kidney and liver and nervous system, and the oedema of the subcutaneous tissues. Whether these diffuse changes occur simultaneously in gradually increasing intensity, or whether they follow a definite sequence in their development, we have so far no means of knowing.

In the majority of cases, where the complex of changes culminates in an eclamptic seizure, the clinical picture is completed before or during labour. In otler instances, however, the end stages are only enacted after birth, and the convulsions, coma, etc., develop some hours after the escape of the uterine contents.

Post-partum eclampsia constitutes a difficulty that must be candidly faced by the research worker; and it has long been apparent to us that an understanding of this variant of the disease would probably throw new light on the ultimate nature and origins of the toxaemia. Anomalies are often of greater value than the commonplace features in directing attention to the underlying facts of a disease,

In a preceding communication we tentatively suggested that a piece of dying placenta retained in the uterus might account for the puerperal seizures. This idea we no longer hold, and we are not in a position to offer any other explanation that we can support with proof. We would direct attention, however, to some interesting analogies which may throw some light on the cause of delay in the manifestation of the symptoms in a post-partum seizure.

There are not a few toxaemias in which a definite interval is known to exist between the introduction of the poison and the development of the symptoms. In chloroform poisoning the phenomena of toxaemia may not develop for a day or two. But a more interesting analogy is phosphorus poisoning. Here the introduction of a toxic dose may lead to immediate signs of irritation. These pass off, and not till forty-eight hours later do the major evidences of toxaemia develop amongst which convulsions may be present. The analogy between the effects of these chemical poisons and eclampsia is brought home in a striking manner by a study of the tissue changes. In both of the chemical poisons massive damage to the liver occurs, and, in delayed chloroform poisoning, the liver changes may closely simulate those present in eclampsia.

It is impossible to aroid the inference that the immediate cause of the symptoms in such cases is the flooding of the system with the breaking-down products of the liver cells (and perhaps of other organs as well), which were killed at the time of the introduction of the poison, and which in their turn, after a varying but considerable period, generate a new and different poison.

We hold that a conception of this sort is reasonable, and it suggests that eclampsia, as we know it, is possibly not the manifestation of the immediate results of the placental poison, but it is dependent on the flooding of the mother's system with the fission products of the cells of the liver (and perhaps of other organs). It is, as it were, a secondary toxaemia that requires some hours or even days for its culmination. If this explanation be correct, it accounts for the occasional delay of the end stages of the toxaemia till the post.partum period.

The analogy of the toxic effects of damaged muscle, which we have referred to on a preceding page, is even more suggestive than that drawn from a study of the chemical poisons. The actual products eliminated from the dying tissues of the leg probably resemble those produced in the placenta. As the researches of Delbet and others show, the lethal result in the former case may be delayed for some time, and the animal eventually succumbs to the effects of the disintegration of the liver and suprarenal glands.

Treatment of Eclampsia and Albuminuria.

There are some suggestions with regard to the treatment of the toxaemias of pregnancy which emerge from this research.

1. Separation of the placenta and retroplacental thrombosis are in some cases, and perliaps in the majority of cases, the factors underlying placental degeneration. Mechanical influences, strain, undue exertion, etc., undoubtedly predispose to these occurrences. Rest should therefore form a cardinal part of the treatment of all such cases. In this way an albuminuria may subside because increasing degeneration of the placenta is avoided.

2. Immediate emptying of the aterus is, theoretically, the ideal line of treatment in severe albuminuria and in eclampsia. It is the ideal method, no matter what theory we adopt regarding their origin, for all are agreed that pregnancy is the ultimate cause. In view of the above theoretical considerations, however, it is possible that when major symptoms develop they orve their origin to a poison introduced into the system some considerable time before, and in the interval the placental danger has subsided or disappeared.

Considerations of this kind would account for the very varied results that follow evacuation of the uterus, and they make logical the attitude adopted by most obstetricians, that severe manipulative measures are apt to stir up the toxaemia to dangerous limits, especially by provoking convulsions, which are readily inaugurated by stimulation of the nerrous system. 


\section{Summary.}

1. Eclampsia and pre-eclamptic toxaemia are due to the products of the early degeneration of a piece of placenta whose blood supply has been interfered with.

2. Infarction of the placenta may be due to mechanical detachment of the organ from the uterine wall-for example, in placenta praevia and in some cases of accidental haemorrhage. In other cases it is due to throm. bosis; this is the factor operating in most cases of eclampsia.

3. Where time is allowed for the elaboration of toxic elements from the detached placenta toxaemia develops. In concealed accidental haemorrhage, therefore, toxaemia is conspicuous. Absorption of placental poisons only occurs if and whilst part of the placenta remains attached to the uterine wall.

4. Toxaemia occasionally accompanies placenta praevia for the same reasons.

5. The toxaemia develops after the haemorrhage; it is therefore not the cause of the bleeding in accidental haemorrhage.

6. The major symptoms of eclampsia are probably due to the flooding of the mother's system with the breaking. down products of liver cells (and perhaps of other tissues), which were lilled some hours or days previously by the placental poison. Compare the interval that elapses before the development of symptoms in phosphorus poisoning, etc. A consideration of this sort accounts for the occasional occurrence of post-partum seizures.

7. Recent investigations on shock (Quénu, Delbet, etc.) support by analogy this conception of eclampsia. These observers have shown that the early disintegration products of muscle are toxic. The injection of these substances into an animal may lill at once. On the other hand, the lethal effect may be delayed for some time, in which case it is due to degeneration of the liver and suprarenal glands. The changes induced in the liver in such a toxaemia resemble those found in the liver in eclampsia.

This investigation was carried out at the instance of the Medical Research Council. It was conducted at the Royal College of Physicians' Laboratory, Edinburgh.

\section{CARDIAC PERISTALSIS AND MITRAL STENOSIS.}

BY

D. W. SAMWAYS, M.A., M.D., D.Sc., M.R.C.P., MENTONE, FRANCE.

Is an article entitled "Dilatation of the Heart," published in the British Medical Journal of February 5th, 1921, I showed that the pressure which the cardiac walls can, theoretically, exert on their contents varies inversely as the cube of the diameter of the enclosed chamber. Thus, when a chamber has by contracting reduced its diameter by one-half, its walls could, because of their physical advantages, exert eight times as great a pressure on the enclosed residual contents, and when the diameter has fallen to one-third they could exert twenty-seven times the pressure that was possible at first, the force of contraction being the same throughout.

Not that I imagine that the cardiac walls can fully effect this, as they are not perfect spheres, and they have deficient muscular areas at their valve orifices. They must, however, be physically capable of more or less following the physical laws which govern contracting hollow spheres, and of exerting enormously greater pressures on their residual than their full contents, or, expressed otherwise, of expelling their residual contents with much less effort than their first expelled contents.

The principle to which I am calling attention can be readily appreciated by the purchase of a big rubber air. balloon with thick walls, as sold to children, and of a similar small rubber balloon with thin walls. If theso are mounted on a three-way glass tube and air blown in by the free orifice, only the big balloon will dilate; the small thin balloon will resist the air pressure which dilates the large thick one. If, now, the inflated big balloon be clamped off and the small one separately inflated, and both afterwards connected together, the small thin balloon will blow its contents into the large thick-walled one and inflate it further.

I maintain that there are conditions in the working of the heart in which the thin-walled auricle can similarly force its contents into the thick-walled ventricle when the ventricle is contracting against it, and that the appreciation of this fact is essential to understanding much that is observable in cardiology.

It is universally admitted that the auricle begins its contraction before the ventricle. The auricle, therefore, is becoming a small chamber while the ventricle is becoming a large one, through receiving the auricular contents. Towards the end of its contraction the auricle has but a few units of area of blood to contract on, while the ventricle, being full at that time, has many. The auricle also by contraction has thickened, while the full ventricle is at its thinnest. Should the contractions overlap, and the ventricle begin its contraction before the auricle has finished, the supposition of so many writers that the ventricle must in that case overcome the auricle is not evident. In the Bramah press it is not the big piston which overcomes the little one, but the reverse; and the full ventricle is at a greater relative disadyantage than the big Bramah piston, having a minimum of contracting efficiency at the moment when the area of resistance is at its maximum.

Now it is commonly supposed that the contraction of the auricle is over before that of the ventricle begins. This view does not accord with that of many of the most noted earlier observers who specially investigated the subject. Thus Dr. Barclay wrote: "The fibres forming the auricle being intimately associated with those which surround the ventricle, the two cavities do not contract and dilate alternately, but the contraction of the one overlaps to some extent that of the other." He added, however : "When the mitral valve is much altered, the more powerful contraction of the ventricle must overcome that of the auricle if there exists anything like an opposition between them."

Professor Potain, the greatest French authority during the last half-century, maintained that, normally, the closure of the auricular-ventricular valves corresponded in time with the opening of the aortic valves, and that the contraction of the auricle lasted till that closure. Had he been able to conceive that the auricle could oppose the ventricle, if their contractions overlapped, he would not have been forced to place, as he was, the beginning of the ventricular contraction at the moment, or just before, the aortic valves opened.

Professor Potain heard the murmur of mitral stenosis (so-called "pre-systolic") during the apex beat, and, believing it to be due to the auricle, maintained that the ventricular contraction must begin later. Dr. Dickinson heard it also during the apex beat, and maintained that it must therefore be ventricular-systolic in origin. Thíe trouble with these observers was that they could not imagine that the auricle could drive its blood on in spite of the ventricle if their contractions overlapped, whereas I have shown that the chance of the auricle being physically able to do this is greater than the chance of the ventricle being physically able to oppose it, especially when the auricle is hypertrophied.

In the classic investigations made by Professors Roy and Adami ${ }^{2}$ graphic tracings are given showing the relation in time of the chief cardiac events. Roy and Adami actually placed tambours in the cardiac chambers so that the various pressure changes could be exactly recorded. The auricular contraction, as given by them, was at its maximum when the apex beat was at its maximum, and had not finished, in normal cases, when the aortic valves opened, thus showing a marked overlapping of the auricular and ventricular contractions.

Moreover, in harmony with this, Roy and Adami found that the papillary muscles did not contract to support the auricular-ventricular valves till the moment when the aortic valves opened. This strongly suggests that some other force was supporting these valves during the first part of the ventricular contraction. That force, fairly obviously, must have been the contracting auricle itself; which I maintain was physically competent to do it.

An overlapping of the systoles is probable a priori, for primitive cardiac contraction is peristaltic, and the essence of a peristaltic contraction is that each segment in sequence takes up the contraction and maintains it during the 